\title{
A FINITENESS PROPERTY OF AFFINE ALGEBRAS
}

\author{
HYMAN BASS \\ (Communicated by Maurice Auslander)
}

\begin{abstract}
Let $A$ be a finitely presented reduced commutative $k$-algebra and
\end{abstract} $J$ an ideal in $A$. If $A$ embeds in $A / J$ then $J=0$.

\section{Statement of THE PROBlem}

We consider the following situation that arose in a technical context in [B].

$K=$ a commutative ring.

$A=$ a commutative $K$-algebra.

$J=$ an ideal in $A$.

$f: A \rightarrow A / J$ is an injective $K$-algebra homomorphism.

We want to conclude that $J=0$ under suitable finiteness assumptions.

(1.1) Theorem. Assume:

(FP) $A$ is a finitely presented $K$-algebra; and

(Red) $A$ is reduced (i.e., all nilpotent elements are zero).

Then $J=0$.

The following examples illustrate the necessity of the hypotheses (FP) and (Red).

(1.2) Example. Let $\left(x_{n}\right)_{n \geq 0}$ be a sequence of indeterminates, $A=K\left[\left(x_{n}\right)_{n \geq 0}\right]$, $J=A x_{0}$, so that $A / J=K\left[x_{1}, x_{2}, x_{3}, \ldots\right]$, and define $f: A \rightarrow A / J$ by $f\left(x_{n}\right)=x_{n+1}(\bmod J)$ for $n \geq 0$. Here $A$ is reduced (and finitely related), but not finitely generated. I do not know if one can relax (FP) and require only that $A$ be finitely generated.

(1.3) Example. Let $R=K[x]$, a polynomial ring in one variable. Let $M=$ $R u \oplus R v$, a free $R$-module with basis $u, v$. Put $A=R \oplus M$ with $M^{2}=0$, and let $J=A v=R v$. Then $A / J=R \oplus R u$ with $u^{2}=0$. Define $f: A \rightarrow A / J$ to be the $K$-algebra homomorphism such that $f(x)=x^{2}, f(u)=u$, and $f(v)=x u$ then

$$
f(A)=K\left[x^{2}\right]+K\left[x^{2}\right] u+K\left[x^{2}\right] x u .
$$

Received by the editors October 23, 1989.

1980 Mathematics Subject Classification (1985 Revision). Primary 13A15, 13 E15. 
Since $K[x]=K\left[x^{2}\right] \oplus K\left[x^{2}\right] x$, the above decomposition of $f(A)$ is a direct sum, hence $f$ is injective. Here $A=K[x, u, v] /\left(u^{2}, u v, v^{2}\right)$ is a finitely presented $K$-algebra, but $A$ is not reduced.

The proof of (1.1) is carried out first in the case when $K$ is noetherian, and then concluded by a reduction to the noetherian case.

\section{Proof of (1.1) When $K$ IS NOETHERIAN}

We may assume that $K \subset A$, so $K$ is also reduced. To show that $J=0$ we can localize, and so assume that $K$ is local, say with maximal ideal $m$, and further that $J_{p}=0$ for all primes $p \neq m$ in $K$. It follows then that $J$ is annihilated by some power of $m$, and hence by $m$ itself, since $A$ is reduced:

$$
m J=0 \text {. }
$$

Next we may assume that $J$ is maximal among ideals of $A$ for which there is a $K$-algebra embedding $f: A \rightarrow A / J$. Since $f(A) \cong A$ is reduced the composite $A \stackrel{f}{\rightarrow} A / J \rightarrow A / \sqrt{J}$ is injective, so $J=\sqrt{J}$, by maximality. Write

$$
J=Q_{1} \cap \cdots \cap Q_{r}
$$

as an irredundant intersection of prime ideals. Put

$$
P_{i}=f^{-1}\left(Q_{i}\right) \in \operatorname{Spec}(A) .
$$

The injectivity of $f$ translates into the condition:

$$
P_{1} \cap \cdots \cap P_{r}=0 \text {. }
$$

It follows that all minimal primes of $A$ occur among the $P_{i}$. Reindex so that each minimal prime of $A$ occurs exactly once among the $P_{1}, \ldots, P_{s}$ (some $s \leq r)$. Putting $J^{\prime}=Q_{1} \cap \cdots \cap Q_{s} \supset J$ we then see that the composite $A \stackrel{f}{\rightarrow} A / J \rightarrow A / J^{\prime}$ is injective, so again by maximality, $J=J^{\prime}$. Thus,

$$
\left\{\begin{array}{l}
\text { each minimal prime of } A \text { occurs } \\
\text { exactly once among } P_{1}, \ldots, P_{r} .
\end{array}\right.
$$

Now $f$ induces a $K$-algebra embedding

$$
f_{i}: A / P_{i} \hookrightarrow A / Q_{i}
$$

and it follows that

$$
P_{i} \text { and } Q_{i} \text { lie over the same prime } p_{i} \text { of } K \text {. }
$$

For $i \in E=\{1, \ldots, r\}$ we can, in view of (4), choose $\sigma(i) \in E$ so that $P_{\sigma(i)} \subset Q_{i}$, and hence also, by (5),

$$
p_{\sigma(i)} \subset p_{i}
$$

Let $E_{m}=\left\{i \in E \mid p_{i}=m\right\}$ and $E_{m}^{\prime}=E-E_{m}$. Recall that $m$ is the maximal ideal of $K$ and we have

$$
0=m J=m \cdot\left(Q_{1} \cap \cdots \cap Q_{r}\right)
$$


It follows from $\left(1^{\prime}\right)$ that $Q_{i}\left(i \in E_{m}^{\prime}\right)$ are the minimal primes of $A$ not containing $m$. Consequently

$$
Q_{i}=P_{\sigma(i)} \quad \text { for } i \in E_{m}^{\prime}
$$

and, therefore, since (2) is irredundant

$$
\sigma\left(E_{m}\right) \subset E_{m} .
$$

Case 1. $E_{m}=E$. Then $m=\bigcap_{i} p_{i} \subset \bigcap_{i} P_{i}=0$, so $K$ is a field. For a finitely generated $K$-algebra $B$ put $t(B)=\sum_{p} \operatorname{transc} \operatorname{deg}_{K}(B / P)$, where $P$ ranges over the minimal primes of $B$. Then we have $t(A / J) \leq t(A) \leq t(A / J)$, the latter because of the embedding of $A$ into $A / J$. Hence $t(A / J)=t(A)$. Since $A$ is reduced it follows easily that $J=0$, whence our result in this case.

Case 2. $E_{m} \neq E$. It follows from (8) that

$$
L=\bigcap_{i \in E_{m}} P_{i} \subset J^{\prime}=\bigcap_{i \in E_{m}} Q_{i}
$$

Hence $f$ induces a $K$-algebra embedding

$$
A / L \rightarrow A / J^{\prime}=(A / L) /\left(J^{\prime} / L\right) .
$$

By induction on $r=\operatorname{Card}(E)$ we conclude that $J^{\prime} / L=0$, that is $J^{\prime}=L$ and therefore, by irredundancy,

$$
Q_{i}=P_{\sigma(i)} \text { for all } i \in E_{m} .
$$

Combined with (7), this gives $Q_{i}=P_{\sigma(i)}$ for all $i \in E$; hence $\sigma: E \rightarrow E$ is a permutation, and $J=\bigcap_{i} Q_{i}=\bigcap_{i} P_{i}=0$.

In applying induction on $r$, we need to check the initial case, $r=1$ when $J=Q_{1} \supset P_{1}=0$. Since $m J=0$ either $m \neq 0$, so $J=0 \quad(A$ is an integral domain) or $m=0$, and we are as in Case 1 above.

\section{REDUCTION TO THE NOETHERIAN CASE}

Write $A=S / L$ where $S=K\left[x_{1}, \ldots, x_{n}\right]$ is a polynomial $K$-algebra, and $L=\sum_{i=1}^{m} S g_{i}$ is a finitely generated ideal. We have $J=J_{1} / L$ for some ideal $J_{1}$ of $S$. The $K$-algebra embedding

$$
f: A=S / L \rightarrow A / J=S / J_{1}
$$

sends $\left(x_{i} \bmod L\right)$ to $\left(p_{i} \bmod J_{1}\right)$ for some $p_{i} \in S(i=1, \ldots, n)$. We first treat:

Case 1. $J_{1}$ is a finitely generated ideal.

Suppose $J_{1}=\sum_{i=1}^{r} S h_{i}$. Let $K^{\prime}$ be a "large" finitely generated (hence noetherian) subring of $K$ so that $S^{\prime}=K^{\prime}\left[x_{1}, \ldots, x_{n}\right]$ contains all $g_{i}, h_{i}$, $p_{i}$. Put $L^{\prime}=\sum_{i=1}^{m} S^{\prime} g_{i}$ and $J_{1}^{\prime}=\left(\sum_{i=1}^{r} S^{\prime} h_{i}\right)+L^{\prime}$. Since $f$ is well defined we have

$$
g_{i}\left(p_{1}, \ldots, p_{n}\right) \in J_{1} \quad(i=1, \ldots, m) .
$$


After enlarging $K^{\prime}$, if necessary, we obtain

$$
g_{i}\left(p_{1}, \ldots, p_{n}\right) \in J_{1}^{\prime} \quad(i=1, \ldots, m) .
$$

Thus we have a well-defined $K^{\prime}$-algebra homomorphism

$$
f^{\prime}: A^{\prime}=S^{\prime} / L^{\prime} \rightarrow S^{\prime} / J_{1}^{\prime}=A^{\prime} /\left(J_{1}^{\prime} / L^{\prime}\right) .
$$

Let $\operatorname{Ker}\left(f^{\prime}\right)=H / L^{\prime}$, where the ideal $H$ of $S^{\prime}$ is finitely generated, since $S^{\prime}$ is noetherian; suppose $H=\sum_{i=1}^{t} S^{\prime} u_{i}$. Since $f=K \otimes_{K^{\prime}} f^{\prime}$ is injective we have $u_{i} \in L \quad(i=1, \ldots, t)$. By enlarging $K^{\prime}$ again we can make $u_{i} \in L^{\prime}$ $(i=1, \ldots, t)$, and render $f^{\prime}$ injective.

Now by the noetherian case of $(1.1)$ we conclude that $J_{1}^{\prime} / L^{\prime}=0$, that is $J_{1}^{\prime}=L^{\prime}$. Since $J_{1}=S \cdot J_{1}^{\prime}$ we conclude also that $J_{1}=L$, hence $J=0$, as we set out to show.

To finish the proof we now make a reduction to Case 1. Let $f(A)=S_{1} / J_{1}$, where $S_{1}$ is a sub- $K$-algebra of $S$. Since $A$ is finitely generated we can choose a finitely generated $K$-algebra $S_{1}^{\prime} \subset S_{1}$ that projects onto $S_{1} / J_{1}$. Putting $J_{1}^{\prime}=$ $J_{1} \cap S_{1}^{\prime}$ we then have $A \cong f(A)=S_{1}^{\prime} / J_{1}^{\prime}$. Since $A$ is finitely presented and $S_{1}^{\prime}$ is finitely generated (as $K$-algebras) it follows that $J_{1}^{\prime}$ is a finitely generated ideal of $S_{1}^{\prime}$. Thus $\left(J_{1}^{\prime} \cdot S\right)+L$ is a finitely generated $S$-ideal. Let $J_{2}$ be any finitely generated $S$-ideal such that $\left(J_{1}^{\prime} \cdot S\right)+L \subset J_{2} \subset J_{1}$. Since $J_{1} \cap S_{1}^{\prime}=J_{1}^{\prime}$ it follows that $J_{2} \cap S_{1}^{\prime}=J_{1}^{\prime}$ also. Hence the image of $S_{1}^{\prime}$ in $S / J_{2}=A /\left(J_{2} / L\right)$ is isomorphic to $S_{1}^{\prime} / J_{1}^{\prime} \cong A$. Thus we can apply Case 1 to $A$ with $J=J_{1} / L$ replaced by $J_{2} / L$, and conclude that $J_{2}=L$. However, we are free to enlarge $J_{2}$ by adding any new generator from $J_{1}$. Thus we see that $J_{1}=L$, that is $J=0$, as we set out to confirm.

\section{ACKNOWLEDGMENT}

I am grateful to Craig Huneke for some helpful discussion and for a simplification of my original proof in the noetherian case.

\section{REFERENCES}

[B] H. Bass, Linearizing flat families of linear representations, Topological Methods in Algebraic Transformation Groups, Progress in Math., vol. 80, Birkhäuser Boston, 1989, pp. 5-10.

Department of Mathematics, Columbia University, New York, New York 10027 